\title{
¿Estado plural o multicultural? El caso del Estado Plurinacional de Bolivia
}

\author{
Bolivia: plural or multicultural State? \\ The Case of a Plurinational State
}

\section{¿Estado plural ou Estado multicultural? \\ O Caso do Estado Plurinacional da Bolívia}

José Andrés Díaz-González

Instituto de Estudios en Población

Universidad Nacional

Costa Rica

Recibido: 19/3/2017 Aceptado: 15/9/2017

\section{Resumen}

El presente ensayo revisa el proceso y consecuencias de la declaración de Bolivia como estado plurinacional, con el propósito de determinar si este responde a un modelo de estado plural o multicultural. Se hace una revisión de los conceptos de pluralismo, como proveniente de una tradición individualista y multiculturalismo, como proveniente de una tradición comunitaria. Apoyándose en el trabajo realizado por diversos actores, así como en la revisión de algunas actuaciones del gobierno Bolivariano, se concluye que la

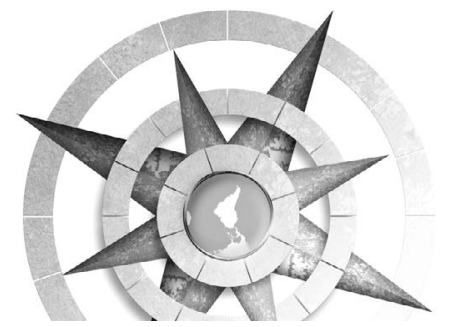

Keywords: Bolivia, State, pluralism, multiculturalism, politics, indigenism
Constitución del 2009 instala un modelo de Estado plural.

Palabras clave: Bolivia, Estado, pluralismo, multiculturalismo, política, indigenismo

\begin{abstract}
This essay reviews the process, and consequences of the declaration of Bolivia as a Plurinational State. Its main purpose is to determine if it responds to a model of Plural or Multicultural State. It development a review of the concepts of Pluralism, as coming from an individualistic tradition; and Multiculturalism, as coming from a community tradition. Based on the work carried out by various actors, as well as the review of some actions of the Bolivarian government, it is concluded that the 2009 Constitution installs a model of Plural State.
\end{abstract}




\section{Resumo}

Este ensaio analisa o processo e as conseqüências da declaração da Bolívia como Estado Plurinacional, com o objetivo de determinar se responde a um modelo de Estado Plural ou Multicultural. Uma revisão dos conceitos de pluralismo é feita, como vem de uma tradição individualista e multiculturalismo, como proveniente de uma tradição comunitária. Baseando-se no trabalho realizado por vários atores, bem como na revisão de algumas ações do governo bolivariano, conclui-se que a Constituição de 2009 instala um modelo de Estado Plural.

Palavras chave: Bolívia, Estado, pluralismo, multiculturalismo, política, indigenismo

En febrero del 2009 se aprueba una nueva constitución en Bolivia, en la cual se reconoce a este país como un Estado Plurinacional, lo cual marca un hito importante en el reconocimiento de la diversidad cultural de los grupos que componen dicha nación, especialmente de las poblaciones indígenas. Sin embargo, esta reforma a la Constitución no es un caso aislado o novedoso; desde la década de 1990 en América Latina empieza a consolidarse, en el ámbito social y político, la necesidad de aceptar que las sociedades latinoamericanas son pluriculturales y multiétnicas; lo cual llevó a que en diversos países de la región se gestaran reformas constitucionales, políticas y educativas con el propósito de reconocer estas características de la sociedad. Sin embargo, estas acciones también han sido criticadas por servir como estrategias de desmovilización de los grupos étnicos, es decir, se convirtió en una parte del discurso utilizado por parte de las clases hegemónicas para dar "respuesta" a las demandas de estos grupos sin producir cambios significativos en la institucionalidad ni, menos aún, abrir espacios para que los integrantes de estos grupos puedan participar de manera efectiva en los procesos de toma de decisiones políticas (Walsh, 2013).

A pesar de estar discutiéndose por cerca de 25 años en la región, la necesidad de reconocer la pluriculturalidad y multietnicidad de las sociedades latinoamericanas, de manera tal que se adopten las medidas jurídicas, políticas e institucionales para el debido reconocimiento $\mathrm{e}$ integración de los distintos grupos étnicos, la realidad es que este proceso dista mucho de estar finalizado (Van Cott, 2005). Es por ello que el presente ensayo revisa el proceso y consecuencias de la declaración de Bolivia como Estado plurinacional, con el propósito de determinar si este responde a un modelo de Estado plural o multicultural.

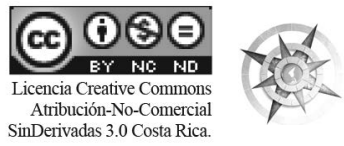


El proceso de globalización que se desarrolla en las últimas décadas del siglo XX e inicio del siglo XXI, no solo produce la integración global de procesos económicos y políticos sino que, además, contribuye a poner de manifiesto que la conformación de las sociedades, lejos de ser homogéneas, son diversas; conformadas por grupos de personas tanto de distintas características, como de variados tamaños, diferentes demandas y diversas necesidades. Esto conlleva a la necesidad de dar respuesta a esta pluralidad por parte de la sociedad, surgiendo dos enfoques diferentes para afrontar las sociedades diversas: el pluralismo y el multiculturalismo. En palabras del politólogo italiano Giovanni Sartori:

Pluralismo y multiculturalismo no son en sí mismas nociones antitéticas, nociones enemigas. $\mathrm{Si}$ el multiculturalismo se entiende como una situación de hecho, como una expresión que simplemente registra la existencia de una multiplicidad de culturas (con una multiplicidad de significados a precisar), en tal caso un multiculturalismo no plantea problemas a una concepción pluralista del mundo. En ese caso el multiculturalismo es sólo una de las posibles configuraciones históricas del pluralismo. Pero si el multiculturalismo, en cambio, se considera como un valor, y un valor prioritario, entonces el discurso cambia y surge el problema. Porque en este caso pluralismo y multiculturalismo de pronto entran en rumbo de colisión (Sartori, 2000, p. 4).

La perspectiva del pluralismo es congruente con la visión del liberalismo, quién lo acepta como algo acertado y positivo en las sociedades democráticas, parte de la premisa que si bien la sociedad está conformada por grupos diversos, cada uno con características particulares, y que deben ser respetados, todos deben ser regulados y cumplir con las mismas condiciones a pesar de sus diferencias. Por su parte, el multiculturalismo también acepta la diversidad como una parte fundamental y necesaria para la democracia, sin embargo, al ser conformada la sociedad por grupos diversos, cada uno con sus propias características y necesidades, acepta que la regulación social se adecue a las particularidades de estos, en otras palabras, busca el reconocimiento de derechos para los grupos minoritarios (Hernández Díaz, Flores Vega, y Espejel Mena, 2008, pp. 367-370).

Otra diferencia entre ambos conceptos es que el pluralismo responde a la tradición liberal individualista, lo que implica que se mantiene la 
prioridad legal y moral del individuo sobre la comunidad; mientras el multiculturalismo responde a la tradición comunitaria, por lo que se da preeminencia a la tradición moral-legal y cultural de la comunidad sobre el individuo (Ulloa Cuellas, 2002, p. 316).

Por lo tanto, el pluralismo es la constatación de la existencia de diferentes culturas en un mismo espacio geográfico y social; sin embargo, estas culturas cohabitan pero influyen poco las unas sobre las otras y no suelen ser permeables a las demás. Por lo tanto, la sociedad que les da acogida suele ser hegemónica y suele establecer jerarquías legales y sociales que colocan a los otros grupos en inferioridad de condiciones; esto puede generar tanto conflictos, como el menosprecio y la creación de estereotipos y prejuicios dificultando la convivencia social, siempre en detrimento de los grupos más débiles. En los casos en que exista equidad y respeto mutuo se puede pasar del pluralismo al multiculturalismo (Argibay, 2003). Debido a lo expuesto hasta el momento, en el siguiente apartado se entrará a analizar con más detalle los conceptos de multiculturalismo y pluralismo, identificando las características de cada uno de estos.

\section{Pluralismo y multiculturalismo ¿en qué se diferencian?}

El pluralismo se basa en la idea de que la diversidad, en el aspecto ideológico, social y político, enriquece la vida pública. Al mismo tiempo, considera que la unanimidad no se da con frecuencia en las relaciones humanas; por lo que se fundamenta en el consenso de las mayorías, sea en el aspecto que sea, para la toma de decisiones, las cuales deben ser aceptadas por todos, aún cuando no sean congruentes con las necesidades y requerimientos de grupos minoritarios (Hernández Díaz et al., 2008, pp. 368-369). Es por ello que el pluralismo es congruente con la idea de la democracia liberal, ya que ambos parten de la idea de que todos deben contar con los mismos derechos y deberes políticos y sociales, sin considerar las diferencias entres los grupos que conforman la población o la manera que se estructura la sociedad, lo cual puede poner en desventaja a los grupos minoritarios que la conforman.

El multiculturalismo no es un hecho social que surge en las sociedades modernas, sino que es una condición normal de toda cultura. Sin embargo, el multiculturalismo en su versión actual está vinculado a dos hechos sociales nuevos. El primero,

54 ¿Estado plural o multicultural?

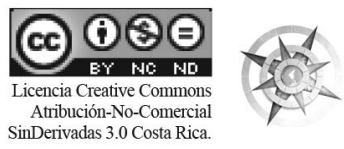


es el reconocimiento de grupos sociales que anteriormente eran invisibles, como por ejemplo las culturas indígenas de América, o los colectivos de gais y lesbianas. A pesar que estos grupos tienen una larga tradición histórica en la lucha por el reconocimiento de sus derechos, es a partir de la década de 1970 cuando estos movimientos inician de forma más sistemática un lento camino a su visibilización y en su constitución como actores sociales colectivos (Cobo, 1999, p. 1).

Así, las ideas en relación al multiculturalismo surgen alrededor de algunos hechos sociopolíticos característicos de la época contemporánea; entre los que se puede mencionar, primero, la descomposición del modelo político basado en el Estado-Nación. Segundo, la sustitución de los principios éticos y políticos ilustrados (igualdad, ciudadanía, derechos humanos, etc.) que han constituido la base moral de la idea moderna de democracia, por el racionalismo instrumental de la economía. Tercero, la visibilización creciente de identidades fuertes, en muchos casos fundamentalistas, que se muestran irreductibles, intolerantes y excluyentes. Esto provoca un fenómeno de debilitamiento de la concepción de sujeto y ciudadanía. Por lo tanto, desde un punto de vista político, es necesario redefinir el concepto de Estado-Nación y su vinculación con la ciudadanía (Cobo, 1999, p. $2)$. Esto debido a que ya no se puede pensar que los Estados albergan una única Nación, es decir, un grupo humano cultural, social, moral y políticamente homogéneo, sino que la sociedad que alberga se compone de una diversidad de grupos, lo que supone el reto de construir un nuevo ordenamiento político que no solo respeta estas diferencias, sino que también las incluya.

Por su parte, Jiménez y Malgesini (2000) indica que el multiculturalismo puede concebirse como un modelo de organización social, que afirma la posibilidad de convivir armoniosamente en sociedad entre aquellos grupos o comunidades étnicas que sean cultural, religiosa o lingüísticamente diferentes. Valora positivamente la diversidad sociocultural y tiene como punto de partida que ningún grupo tiene por qué perder su cultura o identidad propia. En este modelo, la diversidad existente no desaparece sino que se mantiene, se recrea; y no desaparece ni por adquisición de la cultura dominante y abandono del original ni por el surgimiento de una cultura integradora con los aportes de los preexistentes. Por lo tanto, la esencia multiculturalismo se pueden sintetizar en los siguientes puntos: 
1. valoración positiva; por lo que, la organización de la vida en sociedad se realiza sobre bases comunes y respetando las tendencias diferentes así como las complicaciones que ello conlleva.

2. Defensa y reivindicación explícita del derecho a la diferencia, el derecho a ser distinto en valores, creencias, adscripción étnica, etc. Se pone el acento en la diferencia como derecho, al mismo nivel que otras situaciones, por ejemplo de sexo-genero.

3. Reconocimiento general de la igualdad de derechos y deberes (Malgesini y Giménez, 2000).

De esta manera el multiculturalismo "pretende reivindicar una deuda histórica con las culturas minoritarias, que a lo largo de la historia han sido borradas del mapa político y social. Esta corriente busca enmendar el desprecio o falta de reconocimiento de las culturas mediante su acomodo" (Hernández Díaz et al., 2008, p. 638). Así, a diferencia del pluralismo que establece una tolerancia hacia todas las manifestaciones culturales presentes en una sociedad, el multiculturalismo aboga abiertamente por su inclusión en los espacios públicos y en la vida social y política.
De lo anterior se desprende el reto que enfrentan las democracias contemporáneas en relación al pluralismo y al multiculturalismo; ya que, como se ha mencionado, desde una perspectiva de la democracia liberal y representativa, el pluralismo basta para garantizar una igualdad de derechos y deberes a todos los miembros de la población. Pero, si lo que busca es establecerse democracias más abiertas y participativas, la propuesta del pluralismo es insuficiente; en ese caso es necesario recurrir al multiculturalismo como una forma, no solo, de reivindicar los derechos de poblaciones minoritarias que han sido marginadas, también para incluirlas y facilitarles su participación en el espacio público, incluso, realizando acciones afirmativas para facilitar su inclusión, debido a las condiciones de desigualdad en las que se encuentran debido a la estructura social imperante.

\section{El Estado Plurinacional de Bolivia}

El proceso de reconocimiento de Bolivia como Estado Plurinacional, así como sus alcances, impacto e -incluso- contradicciones, ha sido ampliamente analizado por diversos autores (véase: del Pilar Valencia y Egido, 2009; Gustafson, 2009; Regalsky y Breña, 2010; Silber, 2013).
56 iEstado plural o multicultural? El caso del Estado Plurinacional de Bolivia José Andrés Díaz-González
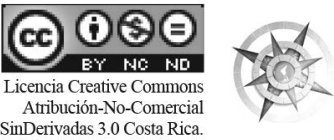
Así, el siguiente apartado se apoya en el trabajo realizado por otros autores, y trata de hacer una reflexión sobre el Estado plurinacional en Bolivia, con el propósito de determinar si este responde a un ideal de multiculturalismo o pluralismo.

Según el Censo Nacional de Población y Vivienda, realizado en el 2012, el 52 \% de la población boliviana se autoidentifica como mestiza, mientras que el $38 \%$ lo hace como indígena y solo el $3 \%$ se considera "blanca" ("El 52 \% de la población se identifica como mestiza", s.f.). Este dato es importante debido a que, primero, ayuda a comprender lo valioso que resulta para la sociedad bolivariana el reconocimiento del Estado como plurinacional y, en segundo lugar, da perspectiva de la inequidad y exclusión que los grupos indígenas han sufrido, históricamente, en dicho país. Como han señalado María del Pilar Valencia e Iván Egido (2009), la intelectualidad boliviana ha mantenido una visión sobre las poblaciones indígenas desde el social-darwinismo; con lo que se buscaba la construcción de lo nacional a partir de la negación del vecino interno: el indígena. En palabras de dichos autores:

En una suerte de europeismo miope se prefería ver como igual al extranjero, antes que al indígena contiguo, a quien se consideraba inferior.

En este contexto, el reconocimiento de los derechos indígenas, siempre estuvo supeditado desde la colonia a la funcionalidad de estos, para los diferentes grupos que ostentaban el poder político-económico (Del Pilar Valencia y Egido, 2009, p. 56).

Dado lo anterior, el triunfo de Evo Morales en las elecciones presidenciales de diciembre del 2005 puede entenderse como un triunfo del indianismo o la revolución indígena. La anterior idea es planteada por Gamboa Rocabado (2010), quién busca alejarse de aquellas posturas, más ortodoxas, que explican la elección de Morales como resultado de una crisis del sistema político boliviano; o bien, de los críticos de la globalización, los cuales visualizan dicha victoria como el nacimiento de un proceso de descolonización, cultural, social y político. Ante estas explicaciones, Gamboa Rocabado recurre al indianismo, el cual considera como una aspiración de reescribir la historia de Bolivia, sobre la base de las visiones culturales de los pueblos indígenas, quienes habían estado sometidos, primero, al yugo español durante la colonia y, posteriormente, por el Estado republicano 
dominado por élites mestizas. Por lo tanto, este discurso político nace tanto como una oportunidad de reinterpretación histórica, como de liberación para importantes sectores de la sociedad boliviana que habían estado históricamente oprimidos (Gamboa Rocabado, 2010, p. 529).

Complementario a lo anterior, el historiador boliviano Hugo Boero destaca que la llegada al poder de Morales debe ser entendida en el marco de un proceso histórico, iniciado en 1952, por medio del cual distintas organizaciones sociales fueron labrando su camino en procura de obtener una mayor cuota de poder político (Boero, 2016). Siguiendo esta idea, Buitrago explica que su elección se debe a diversos factores, desde una campaña política efectiva hasta la mala imagen de las clases políticas dominantes; sin embargo, destaca que uno de los elementos más importantes residió en tener una historia con la cual la gran parte de los bolivianos podía identificarse (Buitrago, 2006, p. 159). Asimismo, este autor señala que otro elemento importante fue el apoyo que tenía de distintas organizaciones de la sociedad civil:

Evo Morales no hubiera podido llegar a la presidencia de Bolivia si no fuese por un esfuerzo concertado de parte de varias organizaciones activistas representando a varios sectores de la sociedad boliviana. Este esfuerzo se llama la Asamblea por la Soberanía de los Pueblos (ASP) y el medio de acción se denomina el Instrumento Político por la Soberanía de los Pueblos (IPSP). La intención es crear un partido político que represente los intereses y preferencias de las organizaciones, y que pueda ser usado para, primero, crear un espacio político y subsecuentemente tomar el poder político del país usando el sistema democrático (Buitrago, 2006, pp. 162-163).

Por lo tanto, la elección de Morales debe ser entendida como el producto de una lucha histórica de una sociedad civil organizada, que trata de tomar las medidas necesarias para adquirir el control del poder político. Lo interesante aquí es que no se trata de una "revolución", sino que la estrategia se basa en utilizar los mecanismos existentes de la democracia formal, para hacerse de control del gobierno y a partir de ahí, generar los cambios que consideren pertinentes. De esta manera, debe verse su gobierno como una señal del reestructuramiento del orden político boliviano (Buitrago, 2006, p. 165).

Lo señalado hasta el momento es importante ya que la elección de Evo

$58 \quad$ iEstado plural o multicultural?

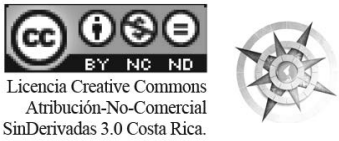


Morales marca un definitivo punto de quiebre en la política bolivariana, al mismo tiempo que es uno de los precursores más importantes para la instalación del Estado plurinacional en esta nación sudamericana. Este se realizó con la aprobación de una nueva Constitución, cuyo trámite inicia en febrero del 2007, y concluye en enero del 2009 con la realización de un referéndum constitucional, por medio del cual Bolivia adquiere una nueva carta magna, el cual contó con la aprobación del 61,43 \% del electorado (Vaca, 2009). Así, posiblemente el cambio más importante en la Constitución del 2009 es el artículo 1 , el cual expresa que:

Bolivia se constituye en un Estado Unitario Social de Derecho Plurinacional Comunitario, libre, independiente, soberano, democrático, intercultural, descentralizado y con autonomías. Bolivia se funda en la pluralidad y el pluralismo político, económico, jurídico, cultural y lingüístico, dentro del proceso integrador del país.

De esta manera, para Gamboa Rocabada la reforma constitucional que declara a Bolivia como Estado Plurinacional es influencia directa del indianismo en dicho proceso político, y tenía el propósito de garantizar las autonomías indígenas, con el objetivo final de doblegar las pretensiones descentralizadoras de las regiones que no respondían al gobierno como Pando, Beni, Santa Cruz, Tanja y Chuquisaca (Gamboa Rocabado, 2010, p. 553). Además, la Constitución del 2009 trae algunas disposiciones con el propósito de empoderar y facilitar el acceso a la población indígena a los puestos de representación política, entre las cuales se destaca:

- Una cuota de parlamentarios indígenas

- Un sistema judicial indígena campesino, al mismo nivel que la justicia ordinaria, junto con un nuevo Tribunal Constitucional plurinacional que tendrá que elegir miembros de los dos sistemas.

- El derecho a la autonomía y el autogobierno indígena, junto con el reconocimiento oficial de sus entidades territoriales e instituciones.

- Propiedad exclusiva de los indígenas de los recursos forestales de su comunidad

A pesar de los importantes avances señalados anteriormente, durante el proceso de discusión y formación del nuevo texto constituyente no todas las propuestas ni demandas de los pueblos indígenas fueron incluidos. En algunos casos los propios pueblos 
indígenas renunciaron a algunas de sus demandas como parte de su estrategia de negociación, con el propósito de asegurar algún otro de sus objetivos; por ejemplo, el control y propiedad de todos los recursos naturales en sus territorios que fue un punto central en las demandas de los pueblos indígenas, sin embargo, fue cedido a cambio de tener el derecho exclusivo de uso de los recursos naturales renovables y ejercer el derecho a la consulta sobre actividades extractivas de los recursos naturales no renovables y la participación de los beneficios de estas actividad (Del Pilar Valencia y Egido, 2009, p. 79).

Asimismo, como señala la socióloga y antropóloga alemana Almut Schilling-Vacaflor, los cambios propiciados en la constitución bolivariana del 2009 significan un importante avance en materia de reconocimiento de los derechos y capacidades de autogestión de los pueblos indígenas en Latinoamérica; sin embargo, han contribuido poco o nada en combatir el autoritarismo y machismo presentes en muchas de las formas organizativas de los pueblos indígenas de Bolivia, lo cual supone un reto a las autoridades políticas:

The recognition of indigenous-campesino self-governing entities and their participation in state politics has the potential to contribute to the building of multiple public spheres. However, many indigenous-campesino systems have authoritarian and sexist characteristics that should not be overlooked. The creation of intercultural institutions is another challenging task for the future that remains largely unresolved. Many social programmes have been implemented, but the degree to which they will bring about long-term structural change is unclear. A difficulty in this context is the immense social pressure to obtain fast, visible results and the MAS party's opportunity to obtain voter support through assistentialist, short-term policies on the one hand and environmental criteria and long-term policies, such as economic diversification, on the other. (Schilling-Vacaflor, 2011, p. 16).

En otras palabras, a pesar que la Constitución de Bolivia del 2009 toma medidas para integrar a la población indígena a la esfera pública, reconociendo al mismo tiempo su autonomía y capacidad de autodeterminación; parece ser que el proceso constituyente dejó de lado la precaria situación que muchas mujeres indígenas-campesinas viven en sus comunidades, por lo que el nuevo texto constituyente no toma ninguna medida especial para solventar

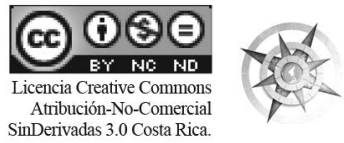


la problemática por la que atraviesa dicho grupo.

A la situación anterior hay que sumarle que, a pesar de lo dispuesto en la nueva Constitución, no desaparecen ni se reducen en intensidad los conflictos entre las autoridades de Gobierno y los pueblos indígenas; esto a pesar de seguir bajo la Presidencia de Evo Morales. Por ejemplo, desde finales del 2012 diversas organizaciones indígenas han mantenido enfrentamientos abiertos con Morales, acusándolo de querer eliminar la autonomía de los grupos civiles indígenas, por medio del uso de prebendas y otras estrategias ligadas al clientelismo político, esto con el propósito de asegurar su apoyo para las elecciones presidenciales del 2014 (López, 2014).

Asimismo, el intento de construir la carretera Beni-Cochabamba, en el 2011, provocó que el gobierno boliviano tuviera que afrontar una serie de movilizaciones y protestas por parte de comunidades indígenas y no indígenas, que se oponían a la construcción de esta carretera; debido a que afectaría una reserva ecológica y forestal, Tipnis, ubicada en el centro del país. El punto más álgido de las movilizaciones devino en una marcha de comunidades indígenas hacia La Paz para hacer llegar su rechazo a la carretera al presidente. La respuesta de las autoridades fue la represión de más de dos mil indígenas que acampaban en la capital boliviana (Díaz Zanelli, 2016).

\section{Consideraciones finales}

El objetivo del presente ensayo era revisar si el modelo de Estado Plurinacional instalado en Bolivia con la constitución del 2009 respondía a un Estado plural o multicultural, si bien dada las limitaciones del documento no puede declarase la respuesta como definitiva, se considera que hay suficiente información para acercarse al modelo al que dicha reforma constitucional responde.

La literatura existente sobre el tema parece acordar que la llegada de Evo Morales a la Presidencia de Bolivia marca un hito sin precedente, siendo esta una valiosa oportunidad para que los pueblos indígenas canalizaran sus demandas históricas, y abogaran por cambios sustantivos en el sistema político, económico y judicial bolivariana, con el propósito de mejorar sus condiciones de vida (Buitrago, 2006; Silber, 2013). Asimismo, como Gamboa Rocabado deja claro, la llegada de Morales al poder puede entenderse como la consolidación del indianismo como proyecto político en Bolivia, el cual 
tiene repercusión directa en el contenido de la constitución del 2009 (Gamboa Rocabado, 2010).

Así, el reconocimiento explícito que la Constitución de Bolivia realiza a la autonomía y capacidad de autoorganización de las poblaciones indígenas, hace indicar que el modelo de Estado en dicho país parece responder al Multicultural, ya que este no solamente establece el respeto por las distintas étnicas, lenguas y culturas existentes en un país, sino que además, abiertamente parece que busca reivindicar la deuda histórica que se ha tenido con los grupos oprimidos, en este caso, la población indígena (Jiménez y Malgesini, 1997, Flores Vega y otros, 2008).

Sin embargo, los conflictos que aún existen entre las autoridades de Gobierno y los pueblos indígenas parecen indicar que el modelo que se implementa es el pluralismo. De esta manera se reconoce que la sociedad bolivariana está constituida por una diversidad de grupos e, incluso, se reconocen los derechos de estos, al punto de promover cuotas de participación electoral para tratar de disminuir las desigualdades existentes al acceso de cargos de autoridad política. Sin embargo, este reconocimiento es principalmente formal, y no trae consigo un cambio radical en la manera en que se ejerce la autoridad o se gestiona el gobierno. Lo anterior se observa claramente en el hecho que las mujeres continúan siendo un grupo relegado, así como en los conflictos que las autoridades de gobierno aún mantienen con los grupos indígenas.

Por lo tanto, en el papel el Estado Plurinacional de Bolivia parece aspirar a un modelo de Estado multicultural, pero en la práctica se acerca más a un Estado pluralista. Esta situación no debe entenderse en ningún sentido como un intento de desvalorar o deslegitimar los esfuerzos que en este país se están llevando a cabo para lograr una mayor integración de los pueblos indígenas; al contrario, se comparte el criterio de diversos autores que exponen que el proceso político boliviano es, en muchos sentidos, un ejemplo para la región latinoamericana, y ha venido a tratar de saldar la deuda histórica que los gobiernos han tenido con estos grupos de la población (Buitrago, 2006; Regalsky \& Breña, 2010; Schilling-Vacaflor, 2011). La afirmación realizada simplemente busca dejar constancia de lo complejo que resulta el desarrollo de un Estado multicultural, y como todo proyecto político no está exento de los problemas y contradicciones.
62 iEstado plural o multicultural?

El caso del Estado Plurinacional de Bolivia José Andrés Díaz-González

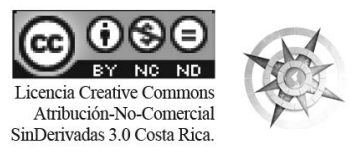


Lo que entonces queda esperar es que el Estado Plurinacional de Bolivia pueda transitar con el tiempo de un modelo pluralista hacia el multiculturalismo al que parece que aspira. Para lograr esta tarea requiere el apoyo de las autoridades políticas, quienes deben respetar y ampliar el marco formal-legal que actualmente posee Bolivia, y el cual es una base importante para lograr un país más inclusivo y solidario para todos los pueblos que lo habitan.

\section{Bibliografía}

Argibay, M. (2003). Multiculturalidad. Recuperado de: http://www.bantaba. ehu.es/formarse/ficheros/view/Exposici\%F3n_2_Sesi\%F3n_1.pdf?revision_id $=34450$ \&package_id $=34415$

Boero, H. (2016, noviembre). Videoconferencia sobre la relación del Estado y la Sociedad en el Gobierno de Evo Morales. Videoconferencia, Universidad de Costa Rica.

Buitrago, M. A. (2006). El significado de la llegada de Evo Morales al poder en la República de Bolivia. Iberoamericana (2001), 6(22), 159-164.

Del Pilar Valencia, M., y Egido, I. (2009). Bolivia: ¿Estado indio? Reflexiones sobre el Estado Plurinacional en el debate constituyente boliviano. Verfassung und Recht in Übersee / Law and Politics in Africa, Asia and Latin America, 42(1), 55-72.

Díaz Zanelli, J. C. (2016, marzo 4). Evo Morales y sus problemas con la población indígena. Servindi - Servicios de Comunicación Intercultural. Recuperado el 4 de diciembre de 2016, de https://www.servindi.org/actualidad-noticias/04/03/2016/evo-morales-y-sus-problemas-con-la-poblacion-indigena

El 52 \% de la población se identifica como mesti$z a$. (s.f.). Recuperado el 3 de diciembre de 2016, de https://www.eldia. com.bo/index.php?c $=\&$ articulo $=\mathrm{El}$ $52 \%$ de la población se identifica como mestiza \&cat $=1 \&$ pla $=3 \&$ \& articulo $=137187$

Gamboa Rocabado, F. (2010). Bolivia frente a su espejo: el indianismo, sus orígenes y limitaciones en el siglo XXI. Estudios Sociológicos, 28(83), 529-560.

Gustafson, B. (2009). Manipulating Cartographies: Plurinationalism, Autonomy, and Indigenous Resurgence in Bolivia. Anthropological Quarterly, 82(4), 985-1016.

Hernández Díaz, A. M., Flores Vega, M., y Espejel Mena, J. (2008). Conciliar el pluralismo y el multiculturalismo mediante la tolerancia. Espacios Públicos, 11(22), 367-379.

López, J. (2014, febrero 5). Evo Morales tropieza con los movimientos indígenas. EL MUNDO. Recuperado de: http:// www.elmundo.es/internacional/2014/02/05/ 52f26126268e3efa738b4571.html

Malgesini, G., y Giménez, C. (2000). Guía de conceptos sobre migraciones, racismo e intercultura/idad. (Segunda Edición). Madrid: Ediciones Catarata.

Regalsky, P., y Breña, M. O. (2010). Political Processes and the Reconfiguration of 
the State in Bolivia. Latin American Perspectives, 37(3), 35-50.

Sartori, G. (2000). Multiculturalismo contra pluralismo. Claves de la Razón Práctica, (197), 4-9.

Schilling-Vacaflor, A. (2011). Bolivia's New Constitution: Towards Participatory Democracy and Political Pluralism? Revista Europea de Estudios Latinoamericanos y del Caribe / European Review of Latin American and Caribbean Studies, (90), 3-22.

Silber, S. (2013a). Refundar Bolivia. La fascinación internacional por Evo Morales y el MAS. Iberoamericana (2001-), 13(50), 197-208.
Silber, S. (2013b). Refundar Bolivia. La fascinación internacional por Evo Morales y el MAS. Iberoamericana (2001-), 13(50), 197-208.

Vaca, M. (2009, febrero 7). América Latina Bolivia promulga nueva Constitución. BBC Mundo. Recuperado en: http://news.bbc.co.uk/ hi/spanish/latin_america/new sid_7877000/7877041.stm

Van Cott, D. L. (2005). From Movements to Parties in Latin America: The Evolution of Ethnic Politics. New York: Cambridge University Press.

Walsh, C. (2013). Políticas y significados conflictivos. Nueva Sociedad, (165), 121-134. 